\title{
Test of a Hybrid Method of Sampling the LGBT Population: Web Respondent Driven Sampling with Seeds from a Probability Sample
}

\author{
Stuart Michaels ${ }^{1}$, Vicki Pineau ${ }^{1}$, Becky Reimer ${ }^{1}$, Nadarajasundaram Ganesh ${ }^{2}$, \\ and J. Michael Dennis ${ }^{3}$
}

\begin{abstract}
This article presents the results of a pilot feasibility study comparing two alternative recruitment approaches based on Respondent Driven Sampling using initial seeds selected from a US nationally representative panel, AmeriSpeak, to augment the number of lesbian, gay, bisexual, and transgender (LGBT) respondents to a short web survey on smoking, discrimination, and health. In the nomination condition after completing the survey both LGBT and non-LGBT seeds were invited to share the names and email address of up to four LGBT persons they knew. In the recruitment condition, seeds were given four unique PINs and links to the survey to distribute to LGBT persons. Both conditions were successful in producing new LGBT respondents. The recruitment condition was much more productive. LGBT seeds (and their recruits) were much connected to and willing to contact other LGBT people they knew to participate in a survey. Comparisons of characteristics and responses from the initial samples and the LGBT referrals as well as comparisons to LGB samples from a large national survey are presented. Results demonstrate the promise of this hybrid technique for increasing the number of LGBT respondents through referrals from an initial probability based sample.
\end{abstract}

Key words: LGBT population; respondent driven sampling; network sampling.

\section{Introduction}

Achieving large representative samples of the sexual and gender minority population is critical to advancing knowledge about these populations, their characteristics, the adversities they face, and potential negative social and health consequences of these adversities (IOM 2011). Before the 1990s, practically all research on lesbian, gay, bisexual, and/or transgender persons used convenience (i.e., nonprobability) samples (e.g., Kinsey et al. 1948, 1953; Bell and Weinberg 1978). With the advent of the HIV/AIDS epidemic, several large probability general population surveys that included questions about sexual orientation, which focused primarily on the sex of sexual partners, were fielded in the United States and in Europe (Laumann et al. 1994; Wellings et al. 1994; Spira et al. 1994). Since then, there has been a slow but steady increase in the number of

${ }^{1} \mathrm{NORC}$ at the University of Chicago, 55 East Monroe Street, Chicago, Illinois 60603, U.S.A. Emails: michaelsstuart@norc.org, pineau-vicki@norc.org, and reimer-becky@norc.org

${ }^{2}$ NORC at the University of Chicago, 4350 East-West Highway, Bethesda, MD 20814, Maryland, U.S.A. Email: ganesh-nada@norc.org

3 NORC at the University of Chicago - AmeriSpeak, 1250 Borregas Ave \#135, Sunnyvale, California 94089, U.S.A. Email: dennis-michael@norc.org 
national large scale probability surveys that include measures of sexual orientation and identity that produce relatively large samples of lesbian, gay, and bisexual persons (IOM 2011). Progress has been slower in the inclusion of measures of gender identity to population surveys, especially at the national level. The relative rarity of LGBT persons, estimated as approximately four percent of the US population (Gates 2014), makes it difficult and quite expensive for most researchers to obtain large representative samples of sexual and gender minority respondents.

This article describes the results of a pilot study of a hybrid sampling approach that combines an implementation of web-based Respondent-Driven Sampling (RDS) with seeds drawn from a probability-based panel of the US population that includes items on sexual and gender identity as part of panel members' profile. We were interested in developing a less expensive alternative to full probability sampling that could be used to generate large enough samples of sexual and gender minority persons to be able to address a wide range of research questions about these populations, including smaller subgroups within the LGBT populations, for example, specific age groups and/or racial and ethnic populations. The goal is to produce as representative a sample as possible, even when resources are limited, that will produce useful answers to important research questions, including the use of nonprobability sampling, what has been referred to as "fit for purpose" (Baker et al. 2013).

Our main goals were: 1) to test a method for generating an oversample of LGBT respondents to augment a sample selected from a national probability sample (specifically the NORC AmeriSpeak national panel) and 2) to assess the quality of the resulting oversample. Schonlau et al. (2014) describes the successful use of web-based RDS to recruit new internet panel members from among friends and acquaintances of an existing representative panel. As far as we are aware, this is the first publication describing an attempt to integrate techniques based on RDS using a probability sample as the starting point to recruit members of a hard-to-survey population (Tourangeau et al. 2014).

RDS is a form of snowball or chain referral sampling developed by Douglas Heckathorn (Heckathorn 1997; Heckathorn 2002; Salganik and Heckathorn 2004) to sample hidden populations that form a single interconnected network starting with a nonprobability, usually purposive sample of "seeds." The assumption that the population of interest is part of a single interconnected network insures that every member of the population has a non-zero probability of being included in the sample. Using network theory and methods, as well as strong assumptions, the goal of classical RDS is to be able to calculate estimates of characteristics of the population being sampled. Central to the method is to trace the chain of referrals, assess the number of potential referrals ("network size"), and generate long referral chains to reduce homophily effects between seeds and referrals. Heckathorn argues that when the assumptions are met, the realized sample is independent of the initial seeds. Most implementations of RDS have been in local areas involving face-to-face interactions, though web-based methods have been used (Wejnert and Heckathorn 2008; Bengtsson et al. 2012; Schonlau et al. 2014; Strömdahl et al. 2015). Each respondent, whether one of the seed cases or their referrals, who completes the survey is asked to refer a set number of other persons they know from among their acquaintances in the 
population of interest. Respondents receive an incentive for their participation and for any of their referrals who successfully complete the survey. This process is repeated iteratively to produce multiple waves of referrals. RDS has been widely used in public health research internationally, in particular in the area of HIV/AIDS research to study hard-to-reach high risk populations such as sex workers, intravenous drug users, and men who have sex with men (McKnight et al. 2006; Bengtsson et al. 2012; Clark et al. 2014; Johnston et al. 2017; Schneider et al. 2017). Under a set of assumptions, and using information on network size and the linkage between respondents and referrals, RDS allows one to generate unbiased estimates of population parameters and generate standard errors for those estimates.

This project borrows the chain referral techniques of RDS for sampling hard-to-reach populations and applies them in a different context. Rather than starting with a set of seeds drawn from a local interconnected network of a hidden population, we apply these techniques to seeds that are drawn from a representative national panel. The initial sample of seeds have known non-zero probabilities of being selected from the target population since they were selected using probability-based methods from the full target population. In this sense, it is also related to network sampling (cf. Sirken 1998; Binson et al. 2007). Our primary focus in this research was not the statistical properties of our estimates, but the feasibility of augmenting the number of LGBT respondents in an efficient and cost-effective manner. In addition, we use this approach to compare the referral cases with seeds that are drawn from a true probability sample and another benchmark survey to make some assessment of how representative the resulting LGBT sample is.

\section{Background}

We conducted two pilot studies to develop and test this new method for sampling the LGBT population. The first pilot is described in more detail in an earlier publication (Pineau et al. 2017), but we give an overview here to provide background for the second pilot, the focus of the present work. These pilots combined aspects of probability sampling and RDS, seeking to take advantage of the strengths of both methodologies in conjunction with sample quality of the AmeriSpeak panel.

In both pilots, we selected a sample of LGBT and non-LGBT panelists from the probability-based AmeriSpeak Panel as seeds for the RDS referral methods. Though RDS does not require a probability-based sample as a starting point, use of a probability-based sample from AmeriSpeak Panel provided a less clustered and more randomly distributed starting point than the purposive sampling used in standard RDS studies. The AmeriSpeak Panel starting sample also provides a basis for evaluating the representativeness of the RDS referred sample on socio-demographic variables by comparing the RDS referred LGBT sample to the probability-based sample of LGBT seeds.

The seed sample of LGBT and non-LGBT panelists were invited to participate in an online survey that included a request to refer LGBT respondents they know to the survey. A brief online survey was developed for both pilots focused on smoking, as this is an area where prior research has found marked differences based on sexual orientation with important health consequences (Lee et al. 2009; Emory et al. 2016). In addition to 
the substantive items about smoking, the survey asked about the number of LGBT persons who are part of their social network for RDS estimation purposes.

In traditional in-person RDS studies, seed sample respondents are given paper coupons to distribute to others in the population of interest so they can participate in the study. Our initial pilot used a variation of this RDS technique, which we refer to as the "nomination" approach. In this approach, seed respondents completed the substantive questionnaire first and then were asked to provide names and email addresses of up to three LGBT persons they knew to whom we would forward an invitation and a link to the survey. We selected this nomination approach at the time to avoid reprogramming our survey software, incorporating a manual process, where research staff added new survey records for the referral cases and sent survey invitations to them as needed.

The results of this first pilot indicated that web-based RDS can produce an LGBT oversample from seeds drawn from the AmeriSpeak Panel. We found that LGBT seeds (and referrals) were much more productive than their non-LGBT counterparts. Seeds and recruits knew many more LGBT friends and family that they said they could refer than the number of email addresses requested in the survey. However, overall the number of seeds (and referrals) providing referrals was disappointing. A total of 146 LGBT and 119 nonLGBT seeds provided 68 referrals (63 from LGBT seeds and five from non-LGBT seeds). None of the referrals nominated by non-LGBT seeds completed the survey, but 23 of the referrals from LGBT seeds did. After two more rounds of dwindling referrals and completes we were able to generate a total of 28 completed LGBT cases, which represented an increase of approximately $20 \%$ additional LGBT cases. Several seed sample respondents, who declined to provide email addresses for LGBT friends and family members in the referral section of the questionnaire, offered comments at the end of the survey indicating that they felt uncomfortable providing contact information for these people without first having the opportunity to get their permission. The comments from respondents in the initial pilot who were hesitant or unwilling to provide referrals led us to believe that allowing respondents to contact their friends directly and without sharing their email addresses with us could be more effective.

Based on these results, we decided to hone the design and carry out a second pilot, the focus of this article. We hypothesized that an alternative recruitment strategy closer to traditional RDS, in which respondents recruit others directly, could substantially increase the size of the final referral sample. Not only is this the standard in traditional RDS, it is also the technique used in web-based RDS surveys of men who have sex with men in Vietnam and Sweden (Bengtsson et al. 2012; Strömdahl et al. 2015).

In the second pilot, we used a split-sample design to compare the nomination approach from the first pilot as one experimental condition to an alternative condition where the panelist seeds were given the survey link to share with friends and family themselves. We also made improvements in the technology for prompting nonrespondents and we increased the number of RDS referrals from three to four. In RDS, the number of referrals is limited in order to create longer referral chains to reduce cumulatively the effects of homophily and fulfill the estimation assumptions. The exact number of referrals is not specified by the theory underlying RDS. It is common to use three, but many RDS studies use more. We settled on a small increase from three to four for the second pilot. The remainder of this article discusses methods and findings from this second pilot. 


\section{Methods}

Data collection for the second pilot survey was conducted between August 18 and September 24, 2017. The source of the probability-based sample of seed cases was AmeriSpeak Panel, NORC's probability-based panel designed to be representative of the US household population. AmeriSpeak Panel was built using a rigorous sampling and recruitment methodology based on probability sampling techniques used by federally sponsored research. During the initial recruitment phase of the panel, randomly selected US households were sampled with known, non-zero probabilities of selection from the NORC National Sample Frame and then contacted by US mail, telephone, and field interviewers (face-to-face). The panel provides sample coverage of approximately $97 \%$ of the US household population. Those excluded from the sample include people with P.O. Box-only addresses, some addresses not listed in the USPS Delivery Sequence File, and some newly constructed dwellings.

\subsection{Sample}

When selecting panelists to act as seed cases for this study, our goal was to have roughly similar size samples of LGBT seeds and non-LGBT seeds. We used panelists' previous reports of LGBT status as a guide and then made a final determination of LGBT status by asking sexual orientation and gender identity (SOGI) questions within the pilot survey. The sample of adults drawn from the panel included a substantial oversample of adults who had previously identified as LGBT, with most of these having previously identified as LGBT in the AmeriSpeak Panel recruitment survey when joining the panel, and some having done so in another previous questionnaire fielded with AmeriSpeak panelists.

These previous self-reports varied in their recency and level of detail. Panel recruitment has occurred on an annual basis since 2014 (when the AmeriSpeak Panel was launched), and panelists are typically asked to complete several panel surveys per month. The SOGI items included in the recruitment survey were added gradually over the past few years starting with the sexual orientation question and then adding the two questions needed to determine gender identity. The questions were chosen with care, and are shown in Appendix A (Subsection 8.1). The sexual orientation question was taken from the National Health Interview Survey, which was developed after extensive cognitive testing by the questionnaire lab at the National Center of Health Statistics for use in general population surveys (Miller and Ryan 2011). The gender identity question selected was based on the recommendations from an experts panel (GenIUSS Group 2014) as used in the California Health Interview Survey.

Due to the changes to the SOGI questions used in the AmeriSpeak Panel recruitment survey over time, the sample included some panelists who had previously answered questions about both sexual orientation and gender identity, some who had answered questions about sexual orientation information but not gender identity, and some whose LGBT status was unknown. For example, it could have included panelists who had only been asked the sexual orientation item in 2014 without updates in the interim years, as well as panelists who had answered the SOGI questions as recently as the months leading up to the second pilot in 2017. Of the total 1,131 panelists sampled, 532 had previously identified as LGBT, 403 had previously identified as non-LGBT, and 196 had an unknown status. The latter were grouped with the non-LGBT seeds. 
Panelists who had participated in the first pilot study were excluded from the sample. The seed sample was also limited to adults age 18 to 55. The decision to limit the age range was made based on other pending work at the time focusing on older LGBT adults. While this choice somewhat limits the generalizability of the experiment, it seemed prudent to avoid overburdening this rare population within the panel. In addition, even though AmeriSpeak is a multi-mode panel that incorporates both web and telephone interviewing, these pilot studies were conducted via web only, purely for budgetary reasons. Over $90 \%$ of the AmeriSpeak Panel responds via the web.

\subsection{Questionnaire}

Separate web surveys were created for the two conditions, which are described in Table 1. The structure of the survey was very similar between conditions, including substantive questions about smoking behavior, social media use, emotional well-being, and perceptions of discrimination, as well as demographic questions, and a request to nominate or recruit LGBT adults to take the survey. The smoking component focused on behaviors related to cigarettes, cigars, and cigarillos, as well as vaping and marijuana.

The demographics section included essentially the same SOGI questions used in previous rounds, shown in Table 1. The panelist seeds received the questions in the order listed here (sexual orientation identity, sex at birth, gender identity), but referral cases received them in a different order $(\mathrm{Q} 14, \mathrm{Q} 15, \mathrm{Q} 13$, i.e., sex at birth, gender, sexual orientation identity).

The structure of the survey differed for the panelist seeds compared to non-panelist referrals who were nominated or recruited, for two reasons. First, demographic

Table 1. Questionnaire.

Q13.

Which of the following best represents how you think of yourself?

1. [IF GENDER1=1 DISPLAY] Gay; [IF GENDER1 $<>1$ DISPLAY] Lesbian or gay

2. [IF GENDER1=1 DISPLAY] Straight, that is, not gay; [IF GENDER $1<>1$ DISPLAY] Straight, that is, not lesbian or gay

3. Bisexual

4. Something else

5. I don't know the answer

Q14.

What sex were you assigned at birth, on your original birth certificate?

1. Male

2. Female

Q15.

How do you describe yourself today?

1. Male

2. Female

3. Transgender

4. I do not identify as male, female, or transgender 
information needed to be collected for referrals, but these items did not need to be asked for panelist seeds since their demographic information was already known. Second, we chose to ask about LGBT status sooner in the survey for referrals than panelists for screening purposes. The questionnaire flows are shown in Appendix B (Subsection 8.2).

For panelist seeds, the questionnaire began with the substantive questions and then asked about sexual orientation and gender identity, to verify any information they had previously provided, and update it if necessary. The majority of panelists who completed the survey provided answers to the SOGI questions that confirmed their previously identified status as LGBT or non-LGBT. Of those who had provided this information in the past, 317 out of 347 (91\%) across the two conditions confirmed their previous status, while $28(8 \%)$ changed their identification from LGBT to non-LGBT, and two (less than $1 \%$ ) changed their identification from non-LGBT to LGBT. A total of 63 panelists who had not answered SOGI questions in the past were sampled for the experiment. Among those, 55 identified as non-LGBT and eight identified as LGBT. In the analyses for this article, we use the responses to the SOGI questions in the survey as described below.

Panelists proceeded past the SOGI questions to an item assessing the size of individuals' LGBT networks. This question is presented in Appendix C (Subsection 8.3). It asked panelists to report the total number of LGBT adults within their social support and communication networks, including family members, friends, acquaintances, co-workers, classmates, and other people in one's community. The network size question is needed for RDS estimation, as persons who have larger networks have a greater chance of being referred to the study. Respondents who said they did not know any LGBT persons did not receive a request to refer others.

After the network size question, panelists were asked to nominate or recruit up to four of the LGBT adults they knew to take the survey. The survey text explained that if others completed the survey, the panelist would receive an incentive themselves, and those they referred to the survey would also receive incentives upon completion. The methods for this request differed between the two conditions and are described below. The last question for panelist seeds was an open-ended field seeking comments about the survey. Panelists received AmeriSpeak points after completing the survey.

When non-panelist referrals entered the survey, the first priority was to assess their LGBT status, to confirm their eligibility. The first few questions asked about basic demographics followed by the SOGI items. Those who identified as non-LGBT were screened out of the survey. Those who identified as LGBT proceeded to the substantive questions, then the network size question, referral request, a screen that collected contact information for an incentive to be mailed to them, and the survey comments.

\subsection{Experimental Conditions}

Sampled panelists were randomly assigned to one of the two experimental conditions: a nomination condition (used in the first pilot) and a recruitment condition. The conditions differed only in the methods by which panelists were asked to refer LGBT friends and family members at the end of the survey.

In the nomination condition, panelists were asked to nominate LGBT adults to participate by providing their names and email addresses. In the recruitment condition, 
they were not asked to provide any contact information and were instead asked to personally invite LGBT adults by directly reaching out to these individuals themselves and sharing a survey link and unique PIN. In both conditions, the referred LGBT non-panelists who completed the survey were also asked to enlist other LGBT individuals to take the survey to generate multiple waves of referrals. The LGBT referrals were invited to refer others using the same technique (nomination or recruitment) as had been used to generate their participation.

A total of 1,131 AmeriSpeak panelists received an invitation to participate in the survey, 565 in the nomination condition and 566 in the recruitment condition. Based on panelist participation rates in previous pilot work, the goal was to have approximately 100 panelist seed cases complete the survey in each of four cells: LGBT nomination, non-LGBT nomination, LGBT recruitment, and non-LGBT recruitment.

\subsubsection{Nomination Condition}

In the nomination condition, the referral item included information about the study's methods and purpose, the importance of the research, confidentiality, and survey incentives. It requested that respondents nominate up to four LGBT people they knew to take the survey by providing their names and email addresses. Respondents who had indicated that their LGBT network size was zero skipped this question. Those who entered information to nominate others to the survey were asked to confirm that the information was correct on the following screen before email invitations were sent.

\subsubsection{Recruitment Condition}

In the recruitment condition, the referral item contained the same wording as the nomination condition about the study background, importance, confidentiality, and incentives. Again, respondents who had indicated that their LGBT network size was zero skipped this question. The item, which is shown in Appendix D (Subsection 8.4), requested that respondents invite up to four LGBT people they knew to take the survey by personally contacting them, rather than by providing their names and email addresses. It provided the survey link and four unique PINs for panelists to pass along to their referrals. It also had a sample survey invitation that they could copy and paste to use along with the unique PINs if desired. In addition, it allowed them to postpone sending invitations until a later, more convenient time by offering the opportunity to receive all of this information in an email for later use.

\subsection{Incentives and Nominations/Referrals}

Each respondent who completed the survey (in either condition) received the equivalent of USD 5.00 for doing so. Panelist seeds received this incentive in the form of 5,000 points, while non-panelist referrals received a USD 5.00 Amazon gift card. Non-panelists were asked to provide their mailing address to receive the gift cards. Those respondents who successfully referred others to the survey received the same incentive again for each referral who completed the survey.

\subsection{Reminders}

Multiple types of participation reminders were sent for this study. In both conditions, panelists who had not yet completed the survey received reminders twice a week. Panelists 
who had completed the survey themselves, but whose referral cases had not yet completed received reminders once a week, to encourage them to remind their friends and family members about the survey. In the nomination condition, we were able to send these types of reminders to referral cases as well, because their email addresses were stored in the questionnaire data. For the recruitment condition, email addresses were not requested, so we were not able to send any reminders to referral cases.

\subsection{LGBT Questions and Definition}

The LGBT definition in this pilot (for both panelists and referral cases) used three questions: first, a question on sexual orientation identity developed for and used on the National Health Interview Survey and the two-step sex/gender identity questions used in the California Health Interview Survey. The exact wording and response categories for these questions is shown above in Subsection 3.2. For a respondent to be considered LGBT, they had to meet one of the following requirements:

- Identify as gay, lesbian, or bisexual at the sexual orientation question

- Identify as transgender on the gender identity question, or as a different sex on the gender identity question than on the sex assigned at birth question

Those who identified as "straight" and reported a gender identity matching their sex assigned at birth were categorized as non-LGBT. Those who reported that their sexual orientation was "something else" or that they did not know how to answer the question, or those who indicated that they did not identify as male, female, or transgender for the gender identity question were also considered non-LGBT.

This relatively restrictive definition of LGBT was made to facilitate the programming of the web survey, which had to determine whether referral cases could proceed to the substantive survey based on their responses to these questions. Mechanisms to allow persons who identify as for example "queer" or "gender non-binary" or some other designations that one might want to include as LGBT could be considered in future applications of this method.

Two panelists declined to answer at least one of the SOGI questions, meaning they had incomplete information, and were categorized as non-LGBT. A total of 11 panelists selected "something else" or "I don't know how to answer this question" for the sexual orientation item and were categorized as non-LGBT. Two of these eleven had a single successful referral to the survey, while the remaining nine had no referrals. Referral cases who fell into this category were screened out of the survey. A total of 19 referrals screened out of the survey as non-LGBT. This included five who identified as cisgender/straight and 14 who selected one of the other categories on the sexual orientation item and/or the gender identity item.

\section{Results}

As in the initial pilot, this pilot was successful in generating an oversample of LGBT respondents via outreach from probability-based panel members and their referrals. A total of 410 panelist seeds (182 LGBT and 228 non-LGBT) and 107 LGBT referrals completed 
the survey. Table 2 summarizes the overall completed seed and referral cases under the two conditions based on whether the panelist seeds had identified themselves as LGBT or not during the survey.

Table 2 shows that the recruitment condition was nearly twice as productive as the nomination condition in yielding completed LGBT referral cases, with a total of 70 completed referral interviews compared to 37 in the nomination condition. This difference is also clear in the visual shown in Figure 1, which displays small squares for each panelist seed case that generated at least one referral, and small circles for each referral interview. Figure 1 details the number of referrals at each stage of the referral process: wave 1 are the referrals from the seeds drawn from the AmeriSpeak Panel, Wave 2 are the referrals made by the completed LGBT referrals in Wave 1, and so on. There are far more referral cases in the bottom half of the figure, representing the recruitment condition, than the top half, which represents the nomination condition.

Figure 1 shows the differences in the two conditions over the iterations of the RDS referral process for the productive LGBT and non-LGBT seeds. In the nomination condition, which required respondents provide a name and email address for referrals, 14 of the 91 LGBT seeds $(15 \%)$ generated a total of 28 referral cases, whereas five of 117 non-LGBT seeds (4\%) generated nine referral cases. Overall, the nomination condition resulted in 37 additional LGBT cases beyond the initial 91, a $41 \%$ increase in LGBT cases. In the recruitment condition where respondents received survey links and PINs to distribute to LGBT friends and family, again 14 of the 91 LGBT seeds (15\%) generated referrals, but these referrals were more productive at each wave than those in the nomination condition. Cumulatively, the recruitment condition resulted in a total of 49 LGBT referrals (compared to only 28 in the nomination condition). In the non-LGBT group in the recruitment condition, eleven of the 111 seeds (10\%) generated a total of 21

Table 2. Comparison of productivity of LGBT and non-LGBT seeds by condition.

\begin{tabular}{|c|c|c|c|c|c|c|}
\hline & \multicolumn{2}{|c|}{ Nomination condition } & \multicolumn{3}{c|}{ Recruitment condition } \\
\cline { 2 - 7 } & $\begin{array}{c}\text { LGBT } \\
\text { sample }\end{array}$ & $\begin{array}{c}\text { Non-LGBT } \\
\text { sample }\end{array}$ & Total & $\begin{array}{c}\text { LGBT } \\
\text { sample }\end{array}$ & $\begin{array}{c}\text { Non-LGBT } \\
\text { sample }\end{array}$ & Total \\
\hline $\begin{array}{c}\text { Total sample } \\
\text { released }\end{array}$ & 268 & 297 & 565 & 264 & 302 & 566 \\
\hline $\begin{array}{c}\text { Completed } \\
\text { panelist seed } \\
\text { interviews }\end{array}$ & 91 & 117 & 208 & 91 & 111 & 202 \\
\hline $\begin{array}{c}\text { Panelists with } \\
\text { at least one } \\
\text { completed } \\
\text { referral }\end{array}$ & 14 & 5 & 19 & 14 & 11 & 25 \\
\hline $\begin{array}{c}\text { Total completed } \\
\text { LGBT referral } \\
\text { interviews }\end{array}$ & 28 & 9 & 37 & 49 & 21 & 70 \\
\hline $\begin{array}{c}\text { Percentage of } \\
\text { additional } \\
\text { completes via } \\
\text { referrals }\end{array}$ & $31 \%$ & $8 \%$ & $18 \%$ & $54 \%$ & $19 \%$ & $35 \%$ \\
\hline
\end{tabular}




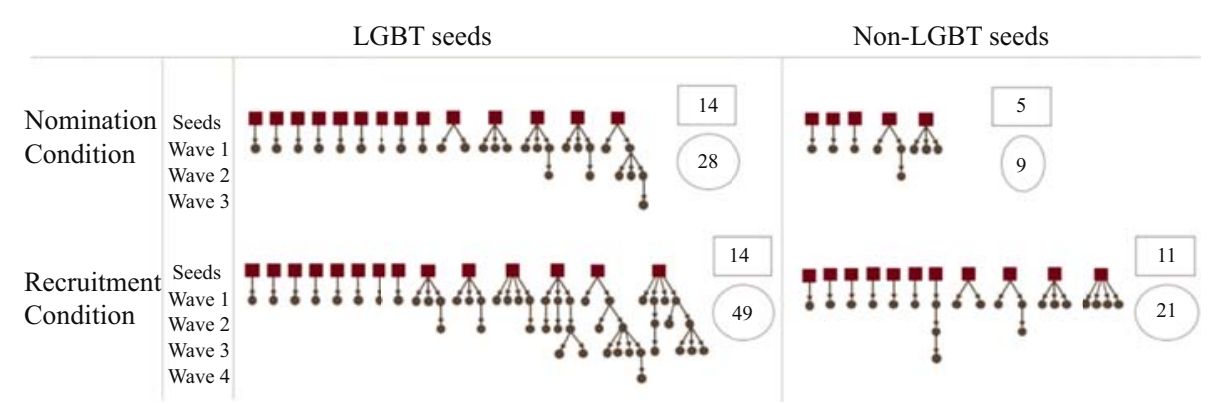

Fig. 1. Comparison of LGBT referrals by wave and condition for productive LGBT or non-LGBT seeds.

referral cases. Overall, the additional 70 LGBT referral completions in the recruitment condition represents a $77 \%$ increase over the initial group of 91 LGBT seeds. The total number of LGBT referral cases in the recruitment condition was higher than the nomination condition for several reasons: more non-LGBT seed cases referred at least one LGBT adult, seeds tended to refer more LGBT adults, and more of the referral cases, in turn, referred additional respondents.

In both conditions, LGBT seeds were more productive than non-LGBT seeds. As seen in Table 2 and Figure 1, independent of condition, the 182 LGBT seeds resulted in 77 LGBT referrals ( 28 from the nomination condition and 49 from the recruitment condition), whereas the 228 non-LGBT seeds only resulted in 30 LGBT referrals (nine from the nomination condition and 21 from the recruitment condition). In part, this may be because LGBT persons know and interact with a larger number of persons who are also sexual or gender minorities. In fact, we find strong evidence of this based on responses to the network size question (see Appendix $\mathrm{C}$ for wording of question).

Table 3 presents the distribution of the responses to the LGBT network size question for the LGBT and non-LGBT panelist seeds, collapsed across the nomination and recruitment conditions. Only $3 \%$ of the LGBT seeds report having no other LGBT people in their close network compared to almost one quarter of the non-LGBT seeds. Almost $60 \%$ of the LGBT seeds have more than ten LGBT people in their network compared to only $20 \%$ of the non-LGBT seeds.

Table 4 compares the actual numbers of successful LGBT referrals for the LGBT and non-LGBT panelist seeds across the two conditions. Even though almost all the LGBT

Table 3. LGBT network size for LGBT and non-LGBT seeds.

\begin{tabular}{|l|c|c|c|c|}
\hline $\begin{array}{l}\text { Number of LGBT } \\
\text { adults known }\end{array}$ & LGBT seeds & Percent & Non-LGBT seeds & Percent \\
\hline 0 & 5 & $3 \%$ & 55 & $24 \%$ \\
\hline 1 & 5 & $3 \%$ & 9 & $4 \%$ \\
\hline 2 & 7 & $4 \%$ & 28 & $12 \%$ \\
\hline 3 & 3 & $2 \%$ & 18 & $8 \%$ \\
\hline $4-10$ & 58 & $32 \%$ & 72 & $31 \%$ \\
\hline $11+$ & 104 & $57 \%$ & 45 & $20 \%$ \\
\hline Total & 182 & & 228 & \\
\hline
\end{tabular}


Table 4. Successful referrals by LGBT and non-LGBT seeds.

\begin{tabular}{|l|c|c|c|c|}
\hline $\begin{array}{l}\text { Number of LGBT } \\
\text { referred }\end{array}$ & LGBT seeds & Percent & Non-LGBT seeds & Percent \\
\hline 0 & 154 & $85 \%$ & 212 & $93 \%$ \\
\hline 1 & 17 & $9 \%$ & 10 & $4 \%$ \\
\hline 2 & 3 & $2 \%$ & 3 & $1 \%$ \\
\hline 3 & 6 & $3 \%$ & 2 & $1 \%$ \\
\hline 4 & 2 & $1 \%$ & 1 & $<1 \%$ \\
\hline Total & 182 & & 228 & \\
\hline
\end{tabular}

seeds know other LGBT persons, $85 \%$ generated no referrals and only $15 \%$ successfully recruited at least one other LGBT person to the survey. Among the non-LGBT seeds, about half as many, 7\%, recruited at least one LGBT respondent. LGBT seeds were also more likely to refer two or more other LGBT respondents.

\subsection{Comparison of Productive and Non-Productive Seeds}

One might ask whether there are demographic differences that account for whether some panelist seeds were productive while others were not. The seeds for this pilot were drawn from the AmeriSpeak Panel, which has a demographic profile of panel members. To address this question, we conducted a binomial logistic regression analysis on whether or not a seed case produced at least one successful LGBT referral by key socio-demographics while controlling for condition and whether or not the seed was LGBT. Table 5 presents the results of the regression. Based on the results from this model, the only variable that was significant at the .05 level was whether the seed was LGBT themselves. Otherwise, none of the demographic variables were significant predictors of seeds' successful recruitment of at least one LGBT referral. It is worth noting that the fact that condition is not significant $(\mathrm{p}=.213)$ may seem surprising, given the large differences in total LGBT

Table 5. Binomial logistic regression on seeds' referral success by demographics and condition $(N=366)$.

\begin{tabular}{|l|r|r|c|}
\hline Dependent variables & \multicolumn{1}{|c|}{ B } & \multicolumn{1}{c|}{ S.E. } & \multicolumn{1}{c|}{ Sig. } \\
\hline Male (ref. female) & -.038 & .342 & .912 \\
\hline African American, non-Hispanic (ref. white/missing) & .174 & .553 & .753 \\
\hline Hispanic (ref. white/missing) & .506 & .395 & .200 \\
\hline Other race, non-Hispanic (ref. white/missing) & -.846 & 1.063 & .426 \\
\hline Some college assoc. degree (ref. high school or less) & -.129 & .859 & .881 \\
\hline Graduate degree (ref. high school or less) & -.564 & .820 & .491 \\
\hline Post-graduate degree (ref. high school or less) & .323 & .844 & .702 \\
\hline Referrer/seed is LGBT (ref. not LGBT) & .902 & .344 & .009 \\
\hline Referral type is nomination (ref. recruitment) & -.416 & .333 & .213 \\
\hline Household size & -.048 & .117 & .683 \\
\hline Income & -.021 & .042 & .616 \\
\hline Age & -.007 & .017 & .691 \\
\hline Constant & -1.823 & 1.129 & .106 \\
\hline
\end{tabular}


referrals by condition described above. However, this regression only looks at seeds and whether they produced at least one LGBT referral or not. As we saw in Figure 1, the overall success of the recruitment condition compared to the nomination condition was due to the fact that seeds in the recruitment condition were more likely to produce multiple referrals and their referrals were also more likely to be productive.

\subsection{Comparing the Resulting LGBT Referral Sample with Probability Sample Benchmarks}

The LGBT seeds were drawn from a national probability sample. Since the seeds can be considered a representative sample of all persons from the US population 18 to 55 years old, they are presumed to also be representative of the LGBT population from the US population 18 to 55 years old. The LGBT referrals are a nonprobability sample generated by an RDS snowball chain-referral sampling process. The ultimate goal is to be able to combine the seed and referral LGBT respondents into a larger, single analytic sample to study the LGBT population and/or compare it to the non-LGBT population. What biases might be introduced by simply combining all the LGBT cases? To investigate this question, we compare the probability and nonprobability components of the LGBT sample to each other and to an independent probability sample of the LGB population in the United States. Unfortunately, there is no national probability survey that asks about gender identity that would allow us to identify LGBT cases. In our sample there were a total of 14 transgender cases: three were seeds in the nomination condition; six referral cases in the nomination condition; and five referral cases in the recruitment condition. There were no transgender seeds in the recruitment condition.

Table 6 compares both the probability sampled Non-LGBT and LGBT seeds and the LGBT referrals from the RDS process from this pilot on key demographic variables. In addition, we provide estimates from the LGB and non-LGB adults, 18 to 55 years old, from the large representative National Epidemiologic Survey on Alcohol and Related Conditions-III (NESARC-III). The NESARC-III is a household-based survey of US adults carried out in 2012-2013 by the National Institute on Alcohol Abuse and Alcoholism. It includes a sexual orientation question that allows one to distinguish lesbian, gay, and bisexual respondents, but no gender identity question. Note that sexual orientation in NESARC-III is based on responses to the question: "Which of the categories on the card best describes you? 1) Heterosexual (straight), 2) Gay or lesbian, 3) Bisexual, 4) Not sure." The wording differs somewhat from the NHIS question used in AmeriSpeak found in Appendix A, which may affect estimates. The percentages presented here are based on weights that take into account the complex sample design. Full documentation of NESARC-III is available on line (National Institutes of Health 2013).

The percentages for the Non-LGBT and LGBT seeds (drawn from the AmeriSpeak Panel) were weighted based on their probabilities of inclusion and adjusting for nonresponse, exclusion of non-web panelists and other non-sampling error via poststratification to socio-demographic population benchmarks. No weights are applied to the LGBT referrals. The percentages in Column D (All LGBT) takes into consideration the adjustments for the seeds described above and no adjustment applied to the referrals by adding the expected number of seeds based on their weighted percentages to the raw count 
Table 6. Comparison of distributions of deeds, LGBT referrals on age, gender, racelethnicity, and education and distributions of LGB and non-LGB respondents from the 2012-2013 NESARC-III general population survey.

\begin{tabular}{|c|c|c|c|c|c|c|}
\hline & $\mathrm{A}$ & B & $\mathrm{C}$ & $\mathrm{D}$ & $\mathrm{E}$ & $\mathrm{F}$ \\
\hline & $\begin{array}{c}\text { Non- } \\
\text { LGBT } \\
\text { seeds }\end{array}$ & $\begin{array}{l}\text { LGBT } \\
\text { seeds }\end{array}$ & $\begin{array}{l}\text { LGBT } \\
\text { referrals }\end{array}$ & $\begin{array}{c}\text { All } \\
\text { LGBT }\end{array}$ & $\begin{array}{c}\text { Gen pop } \\
\text { LGB }\end{array}$ & $\begin{array}{c}\text { Gen pop } \\
\text { Non- } \\
\text { LGB }\end{array}$ \\
\hline Age & & $\mathrm{C}$ & $\mathrm{E}$ & & & \\
\hline $18-34$ & $50 \%$ & $55 \%$ & $77 \%$ & $63 \%$ & $61 \%$ & $44 \%$ \\
\hline $35-49$ & $33 \%$ & $31 \%$ & $18 \%$ & $26 \%$ & $27 \%$ & $39 \%$ \\
\hline $50-64$ & $16 \%$ & $14 \%$ & $4 \%$ & $10 \%$ & $13 \%$ & $17 \%$ \\
\hline $65+$ & $0 \%$ & $0 \%$ & $2 \%$ & $1 \%$ & & \\
\hline \multicolumn{7}{|l|}{ Gender } \\
\hline Male & $50 \%$ & $34 \%$ & $46 \%$ & $38 \%$ & $41 \%$ & $49 \%$ \\
\hline Female & $50 \%$ & $61 \%$ & $42 \%$ & $54 \%$ & $59 \%$ & $51 \%$ \\
\hline Transgender & $0 \%$ & $5 \%$ & $7 \%$ & $6 \%$ & & \\
\hline None of the above & $0 \%$ & $1 \%$ & $5 \%$ & $2 \%$ & & \\
\hline Race/ethnicity & & & $\mathrm{E}$ & $\mathrm{E}$ & & \\
\hline NH white & $58 \%$ & $68 \%$ & $64 \%$ & $66 \%$ & $63 \%$ & $61 \%$ \\
\hline NH black & $13 \%$ & $10 \%$ & $6 \%$ & $8 \%$ & $16 \%$ & $13 \%$ \\
\hline Hispanic & $20 \%$ & $16 \%$ & $24 \%$ & $19 \%$ & $16 \%$ & $18 \%$ \\
\hline $\mathrm{NH}$ other & $10 \%$ & $7 \%$ & $7 \%$ & $7 \%$ & $5 \%$ & $8 \%$ \\
\hline Education & & $\mathrm{C}, \mathrm{E}$ & $\mathrm{E}$ & $\mathrm{E}$ & & \\
\hline Less than HS & $8 \%$ & $6 \%$ & $7 \%$ & $6 \%$ & $11 \%$ & $12 \%$ \\
\hline HS or equivalent & $30 \%$ & $31 \%$ & $7 \%$ & $23 \%$ & $24 \%$ & $25 \%$ \\
\hline More than HS & $62 \%$ & $63 \%$ & $86 \%$ & $71 \%$ & $65 \%$ & $63 \%$ \\
\hline Total Raw N & 228 & 182 & 107 & 289 & 991 & 24,406 \\
\hline
\end{tabular}

Notes: General Population 1 estimates from 18-55 year olds from NESARC-III. Superscripts indicate that the Chi-Square was significant at the .05 level for a variable when compared to the indicated column. E.g., Column B, LGBT Seeds differ significantly from Column C LGBT Referrals. Distribution of C (LGBT Referrals) differs from Column E (LGB in NESARC-III). The absence of a superscript means the Chi-Square was not significant at the .05 level. For example, the Non-LGBT Seeds (Column A) never differed from Column F. The distribution of the LGBT Seeds differed significantly from the distribution of the LGB cases in the general population NESARCIII sample for Education $(\mathrm{p}=.042)$, but did not differ for Age and Race/Ethnicity.

of LGBT referrals. A series of Chi-Square tests based on estimated counts were computed comparing distributions of age, race/ethnicity, and education of seeds with the corresponding weighted distributions obtained in the NESARC-III sample, as well as comparison of the LGBT seeds with the LGBT referrals. Chi-Square comparisons that were significant at the .05 level are indicated by superscripts with the column from which any column differs for the demographic variable. For example, the "C" under column B (LGBT Seeds) for Age indicates that the age distribution of LGBT Seeds and LGBT 
referrals did differ significantly. The LGBT referrals tend to be younger than the seeds. The absence of an "E" under Column B for age, gender, and race/ethnicity indicates that distribution of the LGBT seeds for those variables did not differ from the distribution of the LGB sample in NESARC. On the other hand, the LGBT seeds did differ from the NESARC-III sample on education $(\mathrm{p}=.042)$, with fewer LGBT seeds who had not finished high school than in the NESARC-III sample and more high school graduates.

Overall, the LGBT and non-LGBT (seed) respondents drawn from the AmeriSpeak Panel are quite similar. There are more women among the LGBT than among the nonLGBT panelists. There is a similar tendency in the NESARC-III data. This pattern has also been noted in other recent US surveys, such as the National Survey of Family Growth, in which there are more women than men who report being LGB, especially at younger ages (Chandra et al. 2011). In addition, there is a higher proportion of whites (68\%) among the LGBT seeds than among the non-LGBT seeds (58\%). There is a similar tendency in the NESARC-III data, though not quite as marked. Chi-Square tests comparing the comparable parts of the seeds samples and the general population samples were not significant at the .05 level, implying that one cannot reject the null hypothesis that the samples were drawn from the same distributions.

The sample sizes are quite small, but it is important to note that there does appear to be a greater degree of difference between the probability sample of LGBT panelists and the LGBT referrals than between the LGBT and non-LGBT components of the probability sample. The LGBT referrals are younger, more evenly split between men and women, and more likely to have post-high school education. The LGBT referrals (and the combination of the LGBT seeds and referrals) differ from the national sample of LGB cases from NESARC-III on race/ethnicity and education. The former appears to be due to the smaller proportion of non-Hispanic Black LGBT referrals, which contributes to the significant difference in race/ethnicity when compared to the larger nationally representative sample of LGB cases in NESARC-III. The tendency to refer more educated LGBT cases also produces a skewed distribution on education for both the referrals and the combination of referrals and seed LGBT cases when compared to a national sample of LGB cases.

\section{Summary and Discussion}

Building on an earlier pilot, we carried out a pilot test of two versions of a web-based RDS protocol using seeds drawn from a probability sample panel of the US population. Using samples of identified LGBT and non-LGBT members of the AmeriSpeak Panel, we tested two types of respondent-driven recruiting: a nomination process where respondents were asked to provide a name and email address of people they knew who were LGBT and a referral process that asked respondents to distribute up to four unique PINs to LGBT friends. We found that while both techniques worked, the second approach, which we have called the recruitment condition, was much more effective in generating new LGBT cases. The recruitment condition, starting from the 202 panelist seed sample, split between LGBT and non-LGBT seeds, generated 70 additional LGBT respondents, a $35 \%$ increase. Most of that increase, 49 referrals, was generated by the 91 seeds who were LGBT, which generated an approximately 53\% increase in LGBT cases. This represents a lower bound estimate due to the limited field period for the pilot, which only achieved a maximum of 
four waves. In a longer field period, some of the respondents may have referred other LGBT respondents.

We found strong evidence that a referral process that allows seeds and referrals to carry out the recruitment process on their own has distinct advantages over a nomination process that requires respondents to share their acquaintances' contact information. We surmise that this was largely due to a reluctance on the part of respondents to give out the names and emails of persons they knew to a third party without the accord of those acquaintances, even when given assurances about confidentiality and when there was some monetary incentive for them and for their friends. This recruitment approach is closer to typical RDS and takes fuller advantage of the respondent driven aspect of this sampling technique, which enlists respondents in identifying and recruiting other members of a hidden population of interest (Heckathorn 1997).

The LGBT respondents were much more connected to and willing to contact other LGBT people they knew to participate in a survey. However, it is worth noting that, especially in the recruitment condition, non-LGBT seeds were also successful in recruiting LGBT referrals. In a probability panel that is representative of the general population, the number of LGBT panel members will be small, on the order of four percent, and if one were seeking to scale up the pilot and achieve a larger sample of LGBT cases, one may need to draw both LGBT and non-LGBT members to achieve the number of LGBT referrals sought. This could include a much larger number of non-LGBT than LGBT seeds.

While demonstrating that this method can be used to augment the number of LGBT cases in a resulting analytic sample, the comparisons between the LGBT seeds and the LGBT cases referred and the comparison of the resulting sample with an independent benchmark probability sample of LGB cases did show demographic bias in referrals who tended to be younger and more educated than the seeds. This may be due to younger and more educated LGBT persons being more open and comfortable with their sexual and/or gender minority status, making them more susceptible to be "out" to their friends and acquaintances and more likely to be willing to participate in a survey focused on them. This suggests caution regarding the representativeness of the resulting sample of LGBT persons. While the sample of seeds is a strict probability sample, the final LGBT sample is a combined probability and nonprobability sample. However, we would argue that it has definite advantages compared to a purely nonprobability convenience sample. In addition, the combination of a probability and nonprobability component allows one to measure and potentially adjust for differences in the latter.

\section{Limitations}

There are a number of limitations to this pilot study. For reasons of cost and efficiency, we restricted this implementation to a web-based mode only. We restricted the sample to panelists who prefer to complete surveys on the internet, whereas in usual AmeriSpeak surveys, responding by telephone is also available and is used by just under $10 \%$ of the $18-55$ year olds in the panel. Though weights were applied to the AmeriSpeak seed sample to account for exclusion of the non-web panelists in the seed sample, the differences observed in the comparisons with the household-based NESARC-III data (e.g., age, race, and education) may be due in part to this design choice. Additionally, the characteristics of the referred samples may also be impacted by the exclusion of non-web panelists. 
One of the difficulties in all RDS implementations due to its use of incentives to encourage recruitment is that it also produces an incentive for some respondents to attempt to "game the system" by either attempting to self-nominate or encouraging others to pretend to be eligible to do the survey. These problems are exacerbated in web-based surveys. At the same time, there are techniques to prevent such gaming (e.g., detecting and preventing multiple entries on the same device).

Ultimately, the final sample generated with our hybrid method is a combined probability-based and nonprobability-based sample. There are various methods for weighting and estimation that can be used, such as RDS estimation, propensity weighting approaches, super-population model approaches, and small area estimation approaches. As we continue to test and implement this new hybrid method for sampling rare populations, our intent is to concurrently explore and develop the most appropriate weighting and estimation methods.

\section{Conclusion}

While at one time it was widely believed that it would be impossible to even ask randomly selected respondents about their sexual and gender identity, the existence of a growing number of large representative population surveys that achieve that goal on a regular basis, as well as methods and techniques such as large scale probability panels and innovative nonprobability methods such as RDS prompted us to explore mixing the two in the pilot project presented here. We believe that the results are encouraging and show that this is a promising avenue for producing relatively cost-effective larger samples of LGBT respondents using a hybrid method that mixes probability and nonprobability sampling. More research and experience is needed to improve the methods used here and to better understand the properties of the resulting sample. The results from this pilot clearly indicate that a recruitment approach is preferable to a nomination approach. In future implementations, we would improve the protocol for identifying LGBT respondents to allow persons who are sexual and/or gender minority who use rarer terms to describe their sexual or gender identity, such as queer or gender non-binary to be considered eligible. Future research could also explore methods to increase the effectiveness of the RDS referrals by better explanations of the purpose and importance of recruitment, as well as testing higher incentives while monitoring any signs of increases in gaming the system. In addition, further research is needed on methods for adjusting and combining the nonprobability referral cases with the probability portion of the sample. The approach described here does not necessitate an existing probability panel. It could be implemented with any probability sample by adding an RDS recruitment component, for example, inviting and incentivizing respondents to recruit LGBT acquaintances to participate.

\section{Appendix}

\subsection{Appendix A: Sexual Orientation and Gender Identity Questions}

The sexual orientation and gender identity questions included in the AmeriSpeak registration and recruitment survey have been added over time. Below the variable name 
for each item below, we have noted the years in which these items were included in the survey.

\section{GENDER}

[Asked in 2014 thru early 2017]

Are you ....

1. Male

2. Female

\section{GENDER1}

[Added in 2017]

What sex were you assigned at birth, on your original birth certificate?

1. Male

2. Female

\section{GENDER2}

\section{[Added in 2017]}

How do you describe yourself?

1. Male

2. Female

3. Transgender

4. Do not identify as male, female, or transgender

\section{[IF GENDER2 $=3$ ]}

\section{GENDER3}

\section{[Added in 2017]}

Would you say you are?

1. Transgender, male to female

2. Transgender, female to male

3. Transgender, gender non-conforming

4. Other (please specify)

\section{[IF (GENDER1=1 AND GENDER2=2) OR (GENDER1= 2 AND GENDER2=1)] GENDER4 \\ [Added in 2017]}

Just to confirm, you were assigned $<$ GENDER1 $>$ at birth and now describe yourself as $<$ GENDER2 $>$. Is that correct?

1. Yes

2. No

\section{LGBT}

\section{[Added in 2016]}

This next question is about sexual orientation. Which of the following best represents how you think of yourself?

1. [IF GENDER $1=1$ DISPLAY] Gay; [IF GENDER $1<>1$ DISPLAY] Lesbian or gay

2. [IF GENDER $1=1$ DISPLAY] Straight, that is, not gay; [IF GENDER $1<>1$ DISPLAY] Straight, that is, not lesbian or gay

3. Bisexual

4. Something else

5. I don't know the answer 


\subsection{Appendix B: Questionnaire Flow for Panelists and Referrals}

Panelist Questionnaire Flow

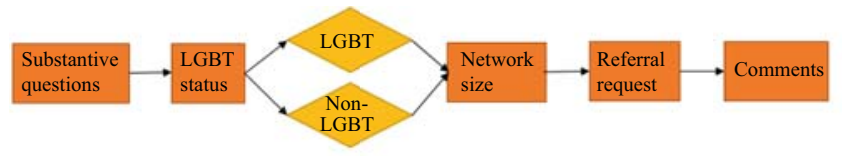

Referral Questionnaire Flow

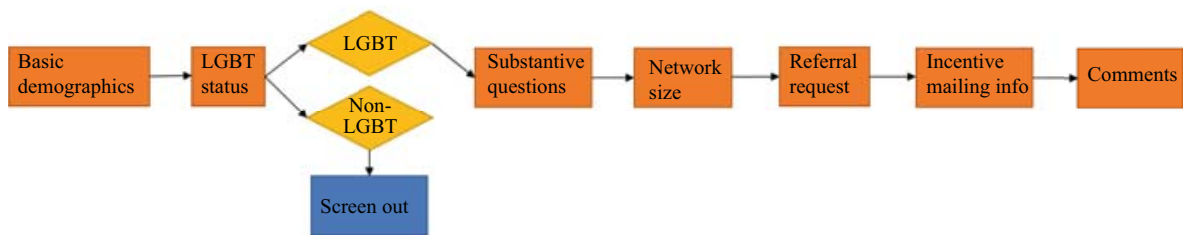

Fig. 2. Panelist questionnaire flow.

\subsection{Appendix C: Network Size Question}

We are interested in learning about people's social support and communication networks. By social support and communication network, we mean the set of other people that each person knows, including family members and friends, with whom they communicate on a fairly regular basis and with whom they exchange information and ideas. For this study, we're particularly interested in the number of people 18 and older who are lesbian, gay, bisexual, or transgender (LGBT) in your social network. Regardless of how you describe yourself, we're interested in the number of LGBT people you know.

If you had to estimate, about how many people 18 or older do you know and communicate with who are lesbian, gay, bisexual, or transgender (LGBT)?

Please include:

- family members

- friends

- acquaintances

- co-workers

- classmates

- other people you know in your community

Please do NOT include:

- people who live outside of the U.S.

- people who you haven't talked to or contacted (via email, text, Facebook, etc.) within the past two years

- people who you no longer have a way of contacting if you wanted to get in touch with them

If you aren't sure of the total number of LGBT people you know, please provide your best guess. 


\subsection{Appendix D: Wording of Referral Request in Recruitment Condition}

We're studying LGBT health and we need your help!

One of the goals of this important health study is to contact and include enough lesbian, gay, bisexual or transgender (LGBT) people to be able to better represent their experiences and compare their experiences with their non-LGBT peers' experiences.

We'd like you to invite [[network=1] the LGBT person you know who is/[network=2-3] any of the $\{\mathrm{NUM}\}$ LGBT people you know who are /[network $>=4]$ up to four of the \{NUM \} LGBT people you know who are] 18 years old or older and who live in the U.S. to take this survey. Each LGBT person you invite to take this survey will receive a USD 5 Amazon gift card for their participation, if they have not already taken the survey. For each person you invite who has not already been invited to take the survey and who successfully completes the survey, you will receive [[AMERISPEAK PANELIST: an additional 5,000 AmeriSpeak points]/[REFERRAL: another USD 5 Amazon gift card]] in addition to the one you will receive for finishing this survey.

In order to receive the maximum of [[AMERISPEAK PANELIST: 20,000 additional AmeriSpeak points]/[REFERRAL: four additional USD 5 Amazon gift cards]], you should invite up to four different LGBT people, and invite only those people who you are sure will take and complete the survey.

The information that the people you invite provide is strictly confidential and will be kept private. We will not associate their names or email addresses with their answers. This information will help us study health patterns in LGBT and non-LGBT populations. If you would like to learn more about this research, click here.

\section{References}

Baker, R., J.M. Brick, N.A. Bates, M. Battaglia, M.P. Couper, J.A. Dever, K.J. Gile, and

R. Tourangeau. 2013. Report of the AAPOR Task Force on Non-Probability Sampling. Oakbrook Terrace, IL: AAPOR. Available at: https://www.aapor.org/AAPOR_Main/ media/MainSiteFiles/NPS_TF_Report_Final_7_revised_FNL_6_22_13.pdf (accessed August 2019).

Bell, A. and M.S. Weinberg. 1978. Homosexualities: A Study of Diversity Among Men and Women. New York: Simon and Shuster.

Bengtsson, L., X. Lu, Q.C. Nguyen, M. Camitz, N.L. Hoang, T.A. Nguyen, F. Liljeros, and A. Thorson. 2012. "Implementation of Web-Based Respondent-Driven Sampling among Men Who Have Sex with Men in Vietnam." PLoS ONE 7(11): e49417. DOI: https://doi.org/10.1371/journal.pone.0049417.

Binson, D., J. Blair, D.M. Huebner, and W.J. Woods. 2007. "Sampling in Surveys of Lesbian, Gay, and Bisexual People." In The Health of Sexual Minorities: Public Health Perspectives on Lesbian, Gay, Bisexual, and Transgender Populations, edited by I.H. Meyer and M.E. Northridge, 375-418. New York: Springer.

Chandra, A., W.D. Mosher, C. Copen, and C. Sionean. 2011. Sexual Behavior, Sexual Attraction, and Sexual Identity in the United States: Data from the 2006-2008 National Survey of Family Growth. Hyattsville, MD: National Center for Health Statistics. 
Available at: https://www.cdc.gov/nchs/data/nhsr/nhsr036.pdf (accessed September 2019).

Clark, J.L., K.A. Konda, A. Silva-Santisteban, J. Peinado, J.R. Lama, L. Kusunoki, A. PerezBrumer, M. Pun, R. Cabello, J.L. Sebastian, L. Suarez-Ognio, and J. Sanchez. 2014. "Sampling Methodologies for Epidemiologic Surveillance of Men Who Have Sex with Men and Transgender Women in Latin America: An Empiric Comparison of Convenience Sampling, Time Space Sampling, and Respondent Driven Sampling.” AIDS and Behavior 18(12): 2338-2348. DOI: https://doi.org/10.1007/s10461-013-0680-0.

Emory, K., Y. Kim, F. Buchting, L. Vera, J. Huang, and S.L. Emery. 2016. "Intragroup Variance in Lesbian, Gay, and Bisexual Tobacco Use Behaviors: Evidence That Subgroups Matter, Notably Bisexual Women." Nicotine and Tobacco Research 18(6): 1494-1501. DOI: https://doi.org/10.1093/ntr/ntv208.

Gates, G.J. 2014. LGB/T Demographics: Comparisons among population-based surveys. Los Angeles, CA: The Williams Institute, UCLA School of Law. Available at: http://williamsinstitute.law.ucla.edu/wp-content/uploads/lgbt-demogs-sep-2014.pdf (accessed April 2018).

GenIUSS Group. 2014. Best Practices for Asking Questions to Identify Transgender and Other Gender Minority Respondents on Population-Based Surveys. Los Angeles, CA: The Williams Institute. Available at: https://williamsinstitute.law.ucla.edu/wp-content/uploads/geniuss-report-sep-2014.pdf (accessed August 2019).

Heckathorn, D.D. 1997. "Respondent-Driven Sampling: A New Approach to the Study of Hidden Populations.” Social Problems 44(2): 174-199.

Heckathorn, D.D. 2002. "Respondent-Driven Sampling II: Deriving Valid Population Estimates from Chain- Referral Samples of Hidden Populations." Social Problems 49(1): $11-34$.

IOM (Institute of Medicine). 2011. The Health of Lesbian, Gay, Bisexual, and Transgender People: Building a Foundation for Better Understanding. Washington, DC: The National Academies Press. Available at: https://www.ncbi.nlm.nih.gov/ books/NBK64795/ (accessed August 2019).

Johnston, L.G., M.M. Mon, M. Steinhaus, and J. Sass. 2017. "Correlates of Forced Sex Among Young Men Who Have Sex with Men in Yangon and Monywa, Myanmar." Archives of Sexual Behavior 46(4): 1001-1010. DOI: https://doi.org/10.1007/s10508016-0761-z.

Laumann, E.O., J.H. Gagnon, R.T. Michael, and S. Michaels. 1994. The Social Organization of Sexuality: Sexual Practices in the United States. Chicago: University of Chicago Press.

Lee, J.G.L., G.K. Griffin, and C.L. Melvin. 2009. “Tobacco Use Among Sexual Minorities in the USA, 1987 to May 2007: A Systematic Review." Tobacco Control 18(4): 275-282. DOI: http://dx.doi.org/10.1136/tc.2008.028241.

McKnight, C., D. Des Jarlais, H. Bramson, C. McKnight, D. Des Jarlais, H. Bramson, L. Tower, A.S. Abdul-Quader, C. Nemeth, and D. Heckathorn. 2006. "Respondent-Driven Sampling in a Study of Drug Users in New York City: Notes from the Field." Journal of Urban Health 83(Suppl 1): 54. DOI: https://doi.org/10.1007/s11524-006-9102-1.

Miller, K.S. and J.M. Ryan. 2011. Design, Development and Testing of the NHIS Sexual Identity Question. Atlanta, GA: Questionnaire Design Research Laboratory. Office of 
Research and Methodology, National Center for Health Statistics. Available at: https://www.impactprogram.org/wp-content/uploads/2011/11/Miller-2011-HHSreport-on-measuring-sexual-orientation.pdf (accessed August 2019).

National Institutes of Health. National Institute on Alcohol Abuse and Alcoholism. 2013. National Epidemiologic Survey on Alcohol and Related Conditions-III (NESARC-III). Available at: https://www.niaaa.nih.gov/research/nesarc-iii (accessed August 2019).

Pineau, V.J., J.M. Dennis, S. Michaels, S. Emery, and N. Ganesh. 2017. "Surveying Rare or Hidden Populations Using a Probability-Based Household Panel.” In Joint Statistical Meetings Proceedings, Survey Research Methods Section, 3733-3746. Alexandria, VA: American Statistical Association. Available at: http://www.asasrms.org/ Proceedings/y2017/files/594086.pdf (accessed August 2019).

Salganik, M. and D.D. Heckathorn. 2004. "Sampling and Estimation in Hidden Populations Using Respondent-Driven Sampling." Sociological Methodology 34: 193-239. DOI: https://doi.org/10.1111/j.0081-1750.2004.00152.x.

Schneider, J.A., B. Cornwell, A. Jonas, R. Behler, N. Lancki, B. Skaathun, S. Michaels, A.S. Khanna, L.E. Young, E. Morgan, R. Duvoisin, S. Friedman, P. Schumm, and E.O. Laumann. 2017. "Network Dynamics and HIV Risk and Prevention in a Populationbased Cohort of Young Black Men Who have Sex with Men." Network Science 5(3): 381-409. DOI: https://doi.org/10.1017/nws.2016.27.

Schonlau, M., B. Weidmer, and A. Kapteyn. 2014. "Recruiting an Internet Panel Using Respondent-Driven Sampling." Journal of Official Statistics 30(2): 291-310. DOI: https://doi.org/10.2478/jos-2014-0018.

Sirken, M.G. 1998. “A Short History of Network Sampling. In Joint Statistical Meetings Proceedings, Survey Research Methods Section, 1-6." Alexandria, VA: American Statistical Association. Available at: http://www.asasrms.org/Proceedings/papers/ 1998_001.pdf (accessed October 2019).

Spira, A., N. Bajos, and the ACSF Group. 1994. Sexual Behaviour and AIDS. Aldershot, England: Avebury.

Strömdahl, S., X. Lu, L. Bengtsson, F. Liljeros, and A. Thorson. 2015. "Implementation of Web-Based Respondent Driven Sampling Among Men Who Have Sex with Men in Sweden." PloS one 10(10): e0138599. DOI: https://doi.org/10.1371/journal.pone. 0138599.

Tourangeau, R., Edwards, B., Johnson, T.P., Wolter, K.M., and Bates, N. (Eds.). 2014. Hard-to-Survey Populations. Cambridge, England: Cambridge University Press.

Wejnert, C. and D.D. Heckathorn. 2008. "Web-Based Network Sampling Efficiency and Efficacy of Respondent-Driven Sampling for Online Research.” Sociological Methods and Research 37(1): 105-134. DOI: https://doi.org/10.1177/0049124108318333.

Wellings, K., J. Field, and A.M. Johnson. 1994. Sexual Behaviour in Britain: The National Survey of Sexual Attitudes and Lifestyles. London: Penguin Books.

Received January 2019

Revised August 2019

Accepted August 2019 